\title{
Design of elastic and dissipation joints in bearing structures of electronic packages
}

\author{
Igor Kovtun ${ }^{1, *}$, Juliy Boiko ${ }^{2}$, Svitlana Petrashchuk ${ }^{1}$, Michat Liss ${ }^{3}$ \\ ${ }^{1}$ Technologies and Design Faculty in Khmelnytskyi National University, Department of Art and \\ Technical Graphics, 11, Instytuts'ka str., Khmelnytskyi, 29016, Ukraine \\ ${ }^{2}$ Faculty of Information Technologies in Khmelnytskyi National University, Department of \\ Telecommunications, Media and Intelligent Technologies, 11, Instytuts'ka str., Khmelnytskyi, 29016, \\ Ukraine \\ ${ }^{3}$ Bydgoszcz University of Life Sciences and Technology, Faculty of Mechanical Engineering, \\ Kaliskiego Street 7, 85-796 Bydgoszcz, Poland
}

\begin{abstract}
Mathematical modeling and experimental research represented in this paper is aimed at dynamic force analysis of circuit cards in order to eliminate or reduce dynamic stress and deflection to an acceptable level and to provide strength and reliability in design of circuit card assemblies subjected to vibration. In conditions when viscous friction forces are negligible and viscous friction dampers, such as liquid dampers, cannot be used reduction of oscillation amplitude in critical frequencies was proposed by creating dry friction damper. On the base of mathematical model, the method for reducing dynamic stress and deflection in critical section of circuit cards in resonance conditions has been developed by inserting additional elastic and dissipation joint introduced by dry friction damper. The dry friction damper device is introduced in three variants of design. The effectiveness of dry friction damper is characterized by its elastic and damping characteristics and especially by the stiffness.
\end{abstract}

\section{Introduction}

Numerous publications devoted to stresses assessment [1] and mechanical strength control [2] for electronic packages, and in particular to printed circuit boards [3], subjected to bending and shear forces [4] and undergoing dynamic deformations [5], performance of dynamic analysis [6] and reliability [7] using vibration reduction design [8] and vibration suppression methods $[9,10]$ indicate of insufficient strength and reliability of modern electronic packages explored in harsh conditions of variety of impacts including mechanical shocks and vibration. Mathematical modeling and experimental research represented in this paper is aimed at dynamic force analysis of circuit cards in order to eliminate or reduce dynamic stress to an acceptable level and to provide strength and reliability in design of circuit card assemblies subjected to vibration.

The previous research published in [11-13] introduced mathematical model for dynamic force analysis of circuit cards (CC). The model described circuit card assembly as the

\footnotetext{
${ }^{*}$ Corresponding author: dr.igorkovtun@gmail.com
} 
separate oscillatory system represented by two-supported weightless prismatic beam with concentrated mass, which creates imbalance and undergoes relative motion in non-inertial frame of reference forced by inertial harmonic oscillation. The initial choice of the beam for simulation is explained by the fact that cylindrical bending of a card can be considered as bending of the set of beam-strips, having rectangular cross-section imaginary cut out of this board in transverse direction, thus the stress and strain calculation of such beam-strips can be performed by using conventional methods applied in strength of materials [14] (internal forces and moments diagrams, stress and strain calculation, etc.).

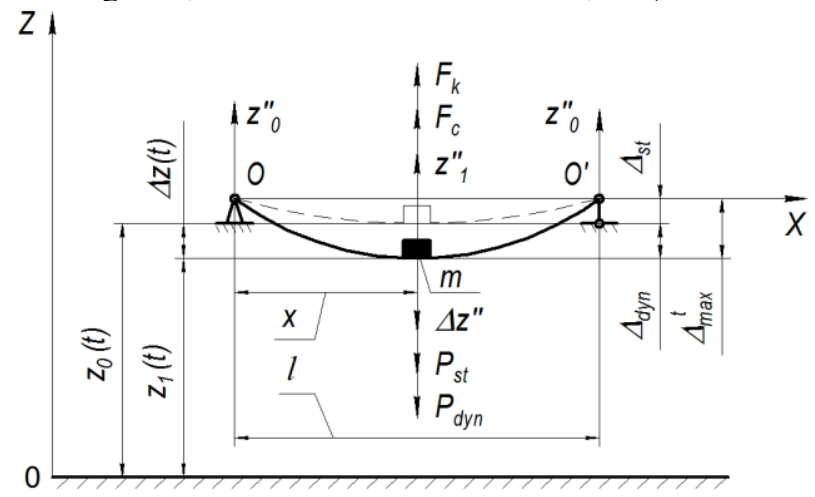

Fig. 1. Oscillatory system: two-supported weightless prismatic beam with single concentrated mass.

The maximal total normal stress (1) and deflection (2), which occur in critical section of $\mathrm{CC}$, are expressed as:

$$
\begin{gathered}
\sigma_{\text {max }}^{t}=\left[Z_{0} \omega^{2} m \frac{1}{\sqrt{\left(1-\frac{\omega^{2}}{\omega_{0}^{2}}\right)^{2}+\frac{4 n^{2} \omega^{2}}{\omega_{0}^{4}}}}+m g\right] x\left(1-\frac{x}{l}\right) \frac{1}{W_{a x}}, \\
\Delta_{\max }^{t} \approx \frac{P\left(3 x l^{2}-4 x^{3}\right)}{48 E J},
\end{gathered}
$$

where $Z_{0}$ - amplitude of oscillation generated by the shaker; $\omega-$ angular frequency, $\omega_{0}-$ natural frequency; $W_{\mathrm{ax}}$ - axial moment of resistance; $x$ - linear coordinate of the mass; $l-$ length of the beam; $E$ and $J$ - Young's modulus and moment of inertia of cross-sectional area of the beam; $P$ - net force representing dynamic force $P_{d y n}=-F_{\mathrm{k}}$ and static force of gravity $P_{\text {st: }}$ :

$$
P=P_{d y n}+P_{s t}=\Delta_{\max }^{t} \delta^{-1}
$$

where $\Delta_{\max }^{t}$ - total deflection produced by the net force $P ; \delta$ - flexibility of the beam in direction of force $P$.

Noteworthy is that strength of CC is recommended to specify with respect to the lowest ultimate strength in the whole assembly, which is represented by soldered joints. So as shown in [15-17] the acceptable stress of CC assembly is defined by ultimate stress of the solder joints calculated with safety factor $\mathrm{s}=2,5$ that compensates errors caused by defects in design and technology.

In conditions of constant dynamic load $P_{0}=Z_{0} \omega^{2} m=$ const and resonance $\omega=\omega_{0}$ functional dependences $(1,2)$ revealed with respect to geometric parameters of $\mathrm{CC}$, in particular to the length $l$ and linear coordinate $x$ of concentrated mass (fig. 2), have provided specification for their acceptable range ensuring strength and stiffness of CC. 


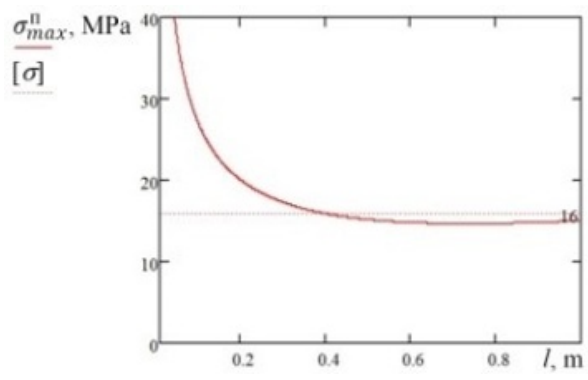

a)

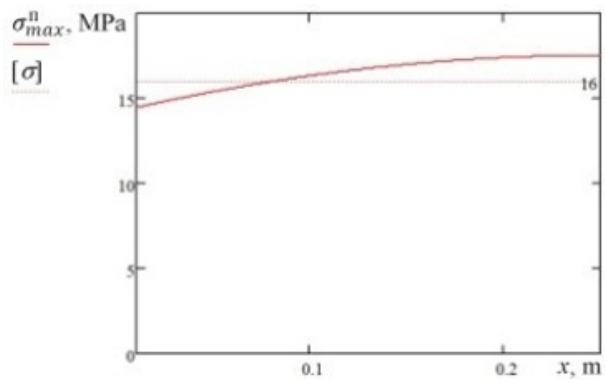

b)

Fig. 2. $\sigma_{\max }^{t}(l)(\mathrm{a}) ; \sigma_{\max }^{t}(x)(\mathrm{b})$ when $w=w_{0}$ and $P_{0}=$ const.

Mathematical model for dynamic stress calculation designed for conditions of resonance and constant dynamic load provided dynamic stress calculation produced by dynamic displacement in relation to static equilibrium position, which does not depend on linear coordinate $x$ of concentrated mass $m$, therefore strength assessment may be performed in engineering calculations with no respect to linear coordinate of the concentrated mass:

$$
\sigma_{d y n}=P_{0} \frac{1}{2 n} \sqrt{\frac{3 E J}{m l}} \frac{1}{W_{a x}} .
$$

\section{Elastic and dissipation joints in the bearing structures}

The previous research published in [18] was aimed at assessing enclosure case design on vibration transmission to circuit cards installed inside.

In this purpose the enclosure case design was varied by changing stiffness of the supporting walls, so three types of enclosure cases were tested: 1) enclosure case with aluminum walls $1 \mathrm{~mm}$ thick; 2) enclosure case with walls of damping material (fiberglass) 1 mm thick; 3) enclosure case with walls removed. Vibration tests have revealed that external damping exceeds internal damping and reducing stiffness of case walls increases vibration transmission from the shaker to CC installed in the cases.

Creation of additional elastic and dissipation joints inside the case [18], such as: 1) banding CCs; 2) bounding rail inserted; 3 ) bounding rail and rubber spacers inserted; and outside the case resulted in reduction of vibration acceleration transmitted to $\mathrm{CC}$ installed inside the case without significant changes in the natural frequency. All types were tested on flexible case suspension of tested case on the shaker. The highest effectiveness was achieved by inserting bounding rail, which more than 4 times reduced maximal vibration acceleration and more than 5 times reduced vibration transmission. Nevertheless the reduced vibration still produced stress and deflection in CCs higher than acceptable strength and stiffness limits and required the further research.

In conditions when viscous friction forces are negligible and viscous friction dampers can not be used (such as liquid dampers) reduction of oscillation amplitude in critical frequencies was proposed by dry friction damper (DFD). Oscillatory system of circuit card with dry friction damper is shown in fig. 3.

With respect to inertial frame of reference, which is represented by supports of the shaker 0 , oscillation of mass $m$ proceeds with acceleration $z_{1}{ }^{\prime \prime}$, due to kinematic excitation generated by oscillations of the beam ends, to which dynamic force is applied and which move with given acceleration $z_{0}{ }^{\prime \prime}$. The absolute acceleration $z_{1}{ }^{\prime \prime}$ of $m$ in the inertial frame of reference may be expressed by relative $\Delta z^{\prime \prime}$ and fictitious $z_{0}^{\prime \prime}$ accelerations as: $z_{1}^{\prime \prime}=-\Delta z^{\prime \prime}+z_{0}^{\prime \prime}$. 


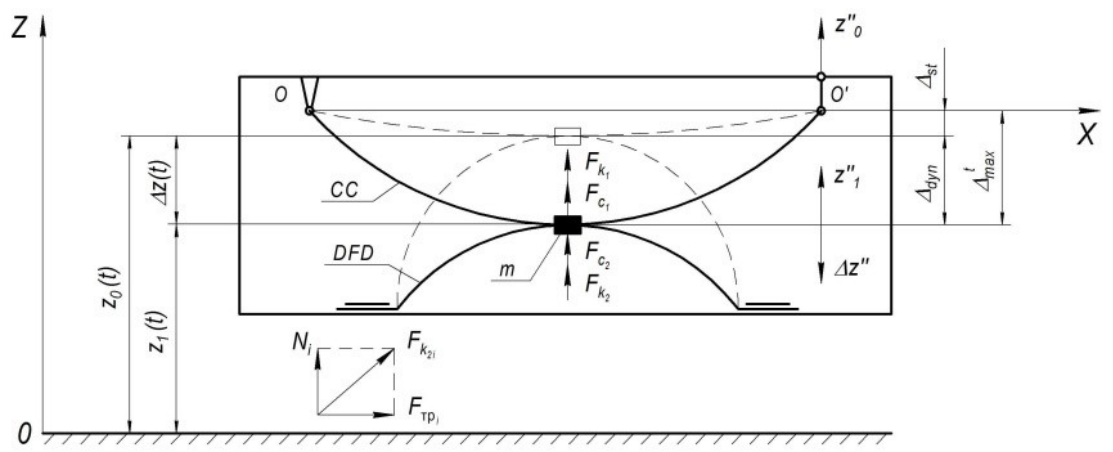

Fig. 3. Oscillatory system of circuit card with dry friction damper.

Then equation of motion in non-inertial frame of reference ( $\mathrm{O}$ and $\mathrm{O}$ ' supports) has the expression analogous to [11]:

$$
m \Delta z^{\prime \prime}+F_{k 1}+F_{k 2}+F_{c 1}+F_{c 2}=m z_{0} ",
$$

where: $m \Delta z^{\prime \prime}$ - relative force of inertia; $m z_{0}{ }^{\prime \prime}$ - fictitious force of inertia; $F_{k_{1}}$ and $F_{k_{2}}$ - elastic forces acting on mass $m$ by CC and DFD correspondently; $F_{c_{1}}$ and $F_{c_{2}}$ - damping forces viscous friction forces in materials of CC and DFD correspondently.

Generalized force of elastic resistance $F_{k_{2}}$ produced by DFD and acting symmetrically on two clutches creates two reactions (fig. 3 ) in each clutch: $N_{i}$ - vertical reaction equal to pressure produced by DFD on the clutch and $F_{f r_{i}}$ - horizontal reaction - friction force, which resists sliding of DFD in the clutch. The net force of resistance in each clutch is expressed as:

Generalized elastic force:

$$
F_{k_{2 i}}=\sqrt{N_{i}^{2}+F_{f i_{i}}^{2}} .
$$

$$
F_{k_{2}}=\sqrt{N^{2}+F_{f r}^{2}}
$$

where $N=\sum_{i} N_{i}$; where $F_{f r}=\sum_{i} F_{f r_{i}}$.

Dry friction force due to negligible dependence on velocity is expressed by Coulomb's formula:

$$
F_{f r}=\mu N
$$

where $\mu$-coefficient of friction; $N$ - pressure.

Substituting (8) into (7) gives:

$$
F_{k_{2}}=N \sqrt{1+\mu^{2}} .
$$

Since pressure DFD produces on the clutch is determined by deflection of DFD $\Delta z$ and its stiffness $k_{2}$, then $N=k_{2} \Delta z$ and (9) becomes:

$$
F_{k_{2}}=k_{2} \Delta z \sqrt{1+\mu^{2}}
$$

Then equation of motion (5) is:

or in brief form:

$$
m \Delta z^{\prime \prime}+k_{1} \Delta z+k_{2} \Delta z \sqrt{1+\mu^{2}}+c_{1} \Delta z^{\prime}+c_{2} \Delta z^{\prime}=m z_{0}^{\prime \prime},
$$

$$
\ddot{q}+q\left(\omega_{01}^{2}+\omega_{02}^{2} \sqrt{1+\mu^{2}}\right)+2 n \dot{q}=Z_{0} \omega^{2} \sin (\omega t),
$$

where $q=\Delta z(t) ; n=\frac{c_{1}+c_{2}}{2 m}-$ total descent rate, where $c_{1}, c_{2}$ - damping coefficients of CC and DFD correspondently; $\omega_{01}=\sqrt{\frac{k_{1}}{m}}$ and $\omega_{02}=\sqrt{\frac{k_{2}}{m}}$ - natural frequencies, where $k_{1}, k_{2}-$ stiffness of CC and DFD correspondently. 
Solution to (11) has expression analogous to [11], where oscillation amplitude, which is maximal dynamic deflection of circuit card $\Delta_{d y n}^{\max }$, proceeds in relation to static equilibrium position of oscillatory system $\Delta_{\text {st }}$ (fig. 3 ) and is expressed as:

$$
\Delta_{d y n}^{\max }=\frac{Z_{0} \omega^{2}}{\sqrt{\left(\omega_{01}{ }^{2}+\omega_{02}^{2} \sqrt{1+\mu^{2}}-\omega^{2}\right)^{2}+(2 n \omega)^{2}}},
$$

When $\omega=\omega_{0}$ :

$$
\Delta_{d y n}^{\max }=\frac{Z_{0} \omega_{0}^{2}}{\sqrt{\left(\omega_{02}^{2} \sqrt{1+\mu^{2}}-\omega_{02}^{2}\right)^{2}+4 n^{2} \omega_{0}^{2}}} .
$$

where $\omega_{0}=\sqrt{\omega_{01}^{2}+\omega_{02}^{2}}$ - natural frequency of the oscillatory system.

The effectiveness of DFD is characterized by its elastic and damping characteristics and especially by the stiffness $k_{2}$. With sufficient accuracy the stiffness of curved DFD can be calculated as for the straight beam. In condition when concentrated mass is assumed centered to CC and DFD stiffness of both bodies ( $i=1,2$ correspondently) are defined by the formula:

$$
k_{i}=\frac{48 E_{i} J_{i}}{l_{i}^{3}} \text {. }
$$

Dynamic deflection of CC is produced by dynamic force $P_{1}=-F_{\mathrm{k} 1}$ :

$$
P_{1}=k_{l} \cdot \Delta_{\text {дин. }}
$$

Maximal internal bending moment corresponds to bending moment produced by $P_{1}$ in the middle cross-section of CC:

$$
M_{1}^{\max }=P_{1} \frac{l_{1}}{4}
$$

In accordance to bending formula [14] function of maximal dynamic stress in CC with respect to stiffness of DFD in resonance condition is:

$$
\sigma_{d y n}^{\max }\left(k_{2}\right)=Z_{0} \frac{k_{1}+k_{2}}{m} \frac{1}{\sqrt{\frac{k_{2}^{2}}{m^{2}}\left(\sqrt{1+\mu^{2}}-1\right)^{2}+4 n^{2} \frac{\left(k_{1}+k_{2}\right)}{m}}} k_{1} \frac{l_{1}}{4} \frac{1}{W_{a x}} .
$$

Function of maximal dynamic deflection (13) of CC with respect to stiffness of DFD in resonance condition is:

$$
\Delta_{d y n}^{\max }\left(k_{2}\right)=Z_{0} \frac{k_{1}+k_{2}}{m} \frac{1}{\sqrt{\frac{k_{2}^{2}}{m^{2}}\left(\sqrt{1+\mu^{2}}-1\right)^{2}+4 n^{2} \frac{\left(k_{1}+k_{2}\right)}{m}}} .
$$

On condition of $P_{0}=$ const provided by constant $\ddot{\Delta z_{0}}=Z_{0} \omega_{0}^{2}$ functions (17) and (18) become:

$$
\begin{gathered}
\sigma_{d y n}^{\max }\left(k_{2}\right)=P_{0} \frac{1}{\sqrt{k_{2}^{2}\left(\sqrt{1+\mu^{2}}-1\right)^{2}+4 n^{2} m\left(k_{1}+k_{2}\right)}} k_{1} \frac{l_{1}}{4} \frac{1}{W_{a x}} ; \\
\Delta_{d y n}^{\max }\left(k_{2}\right)=P_{0} \frac{1}{\sqrt{k_{2}^{2}\left(\sqrt{1+\mu^{2}}-1\right)^{2}+4 n^{2} m\left(k_{1}+k_{2}\right)}}
\end{gathered}
$$

Fig. 4 demonstrates functions (fig. 4 , a) and (fig. 4, b) when $\ddot{\Delta z_{0}}=10 \mathrm{~m} / \mathrm{s}^{2}$. Input parameters for CC were identical to table 1, and parameters of DFD were: dimensions $50 \times 10 \times 1 \mathrm{~mm}$; steel, $E=200 \mathrm{GPa}$, descent rate $n_{2}=0,1 \mathrm{~s}^{-1}$; coefficient of friction $\mu=0,2$. Acceptable stress and deflection found in $[15-17]$ make $[\sigma]=16 \mathrm{MPa}$ and $[\Delta]=4.1 \mathrm{~mm}$. 


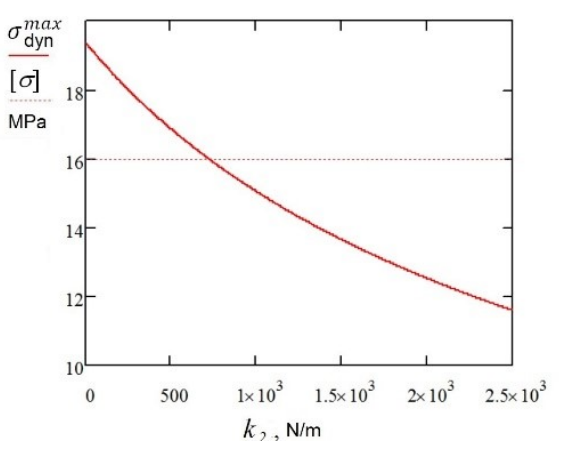

a)

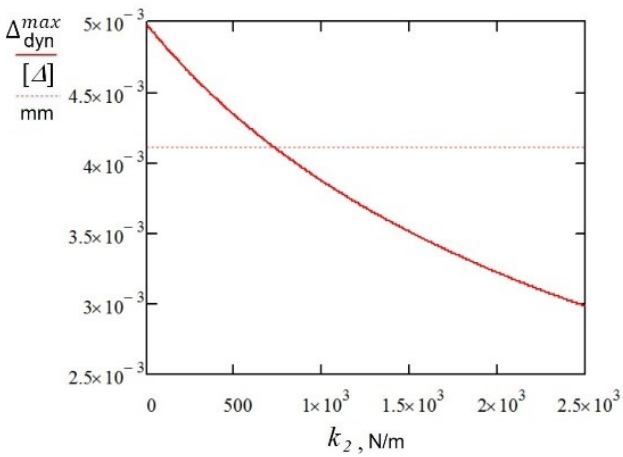

b)

Fig. 4. $\sigma_{d y n}^{\max }\left(k_{2}\right)(\mathrm{a}) ; \Delta_{d y n}^{\max }\left(k_{2}\right)(\mathrm{b})$ when $\omega=\omega_{0}$ and $P_{0}=$ const.

The represented dependences were obtained for the first critical frequency where as shown in [11] stress and strain conditions are characterized by maximal stress and deflection in critical section of CC. As demonstrated by fig. 4 maximal dynamic stress and deflection can be effectively decreased by increasing DFD stiffness.

On the base of mathematical model $(19,20)$ the method for reducing dynamic stress and deflection in critical section of CC in resonance condition has been developed by inserting additional elastic and dissipation joint introduced by dry friction damper whose structure is represented in fig. 5 .

\section{Dry friction damper design specification}

Some of known damping techniques [19-23] propose reducing amplitude of vibration by either increasing stiffness of CC or by damping devices. Increasing stiffness in fixtures of CCs inside the case of enclosure or increasing stiffness of CC structure by using stiffeners is characterized by increased mass or volume and by effects, which do not eliminate oscillations of CCs as they follow oscillations of the enclosure and transmit them to electronic components being damaged as engaged in resonance.

Some damping devices integrate elastic and viscous materials into the $\mathrm{CC}$. However such modification is not applicable to readymade $\mathrm{CC}$. Besides elastic and viscous damping devices are not effective in frequency range higher than $50 \mathrm{~Hz}$, they are temperature dependent and cumbersome.

Another dampers using dry friction provide reducing vibration by flexible bodies (wires) undergoing friction as mounted in between two CCs. However technical specification of such DFDs and assessment of their effectiveness becomes a difficult problem due to their properties and complex geometry.

The DFD represented in this research is introduced by three variants of design (fig. 5). DFD is attached to critical section (in the center) of CC and joined to the case of enclosure by the clutch. The effectiveness to reduce maximal dynamic stress and deflection is provided by its elastic and dissipation properties, in particular by its stiffness. The stiffness of DFD is directly proportional to generalized elastic resistance force $F_{k_{2}}$, it produces, which consists of elastic resistance force $N$ producing pressure of DFD onto the clutch and friction force $F_{\text {fr }}$ that resists damper sliding in the clutch. Increasing mentioned forces with increasing amplitude of vibration provides reducing dynamic stress and deflection transmitted to CC.

In accordance to mathematical model (19) engineering calculation of minimal threshold value for stiffness of DFD $k_{\min }$, which ensures compliance with acceptable strength standard 
$\sigma_{d y n}^{\max } \leq[\sigma]$ for given parameters of CC: $k_{1} ; m ; W_{\mathrm{ax}} ; l_{1} ; n_{1}$; and acting dynamic force $P_{0}$, is performed by formula:

$$
k_{\text {min }}=\frac{\sqrt{4 n^{4} m-\left(\sqrt{1+\mu^{2}}-1\right)^{2}\left(4 n^{2} m k_{1}-\left(\frac{P_{0} k_{1} l_{1}}{4 W_{a x}[\sigma]}\right)^{2}\right)}-2 n^{2} m}{\left(\sqrt{1+\mu^{2}}-1\right)^{2}} .
$$

For the case shown in (fig. 5 , a) $k_{\min }=718.75 \mathrm{~N} / \mathrm{m}$.

Specification of DFD geometric parameters: $l \times b \times h$ is performed by formula (14) for the given elasticity module $E_{2}$ of the used material.

Obviously the effectiveness of DFD is provided as by increasing its stiffness so by increasing descent rate and coefficient of friction, which are determined by material and condition of the friction surfaces: DFD 2 (fig. 5, a, b) or 6 (fig. 5, c) and clutch 4 (fig. 5). However providing and specification of those parameters in real design of $\mathrm{CC}$ becomes a complex problem therefore their minimal reference values [14] are recommended to be used so possible difference in calculation of $k_{\min }$ can used as safety factor.

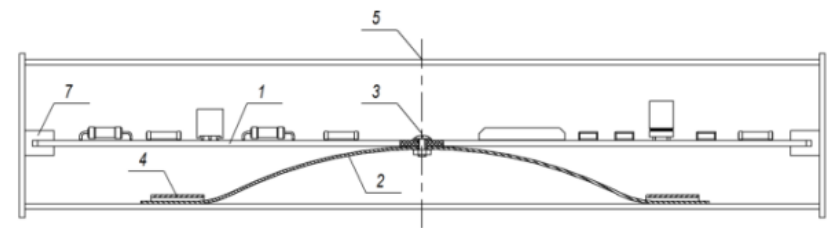

a)

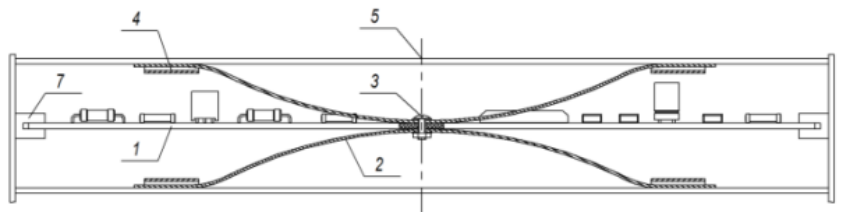

b)

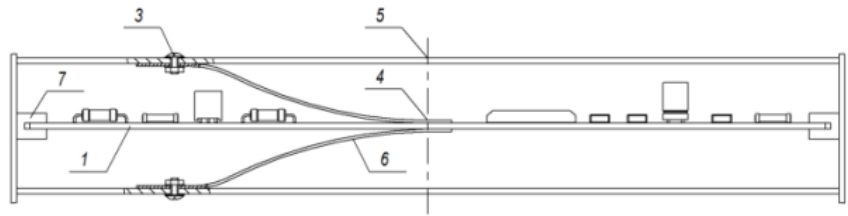

c)

Fig. 5. Dry friction damper structure: a) DFD; b) double DFD; c) half-DFD, where $1-C C ; 2-$ DFD; 3 - fitting; 4 - clutch; 5 - enclosure case; 6 - half-DFD; 7 - CC fixtures.

\section{Experimental verification}

The measuring and test installation (fig. 6) was designed for measurement and analysis of deformations in small-size structural parts and components of electronic packages, while they are subjected to static and dynamic mechanical, thermal and pressure impacts reproducing complex real-world stress and strain conditions [16]. Methods of electrotensometry, acoustic emission, vibration analysis and high-speed video-shooting were implemented. Measuring train consisted of three measuring systems: tensometry 8ANC-26, vibration PI-19 and acoustic emission AF-15 instruments, which are connected to personal computer through the analog-to-digital converter and system interface. Dynamic tests were conducted by the shaker VEDS-200A, static tests were conducted by tension machine IR-5057-50. 
The measuring and testing train has been improved and adapted to current objects of research unlike its traditional usage for large-size objects, which were tested by simple loads in laboratory conditions.

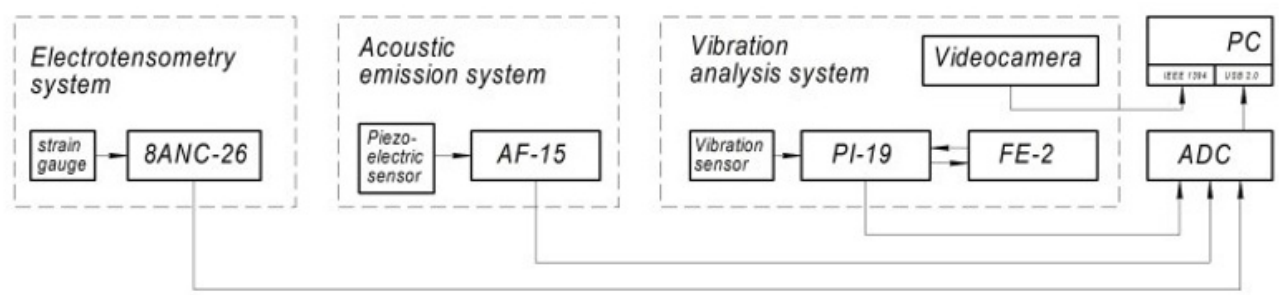

Fig. 6. The measuring and test installation

Verification of analytic estimation is based on determining actual physical and mechanical parameters, which are likely to vary depending on technology, temperature, shape etc. The methods of testing parameters has been applied, in which deflection and deformation, normally calculated, are instead to be found experimentally and considered as given, but physical and mechanical parameters, normally given for mathematical model, are to be determined.

Young's module $E$ and descent rate $n$ were found by testing CC and DFD samples by static middle-point bend test. The force threshold limit was calculated as:

$$
[P]=\frac{2 b h^{2}}{3 l}[\sigma]
$$

where $l \times b \times h-$ dimensions of samples; $[P]=6.67 \mathrm{~N}$.

During static bend test the following parameters were read: force $P$; deflection $\Delta$ and the strain measured by two strain gauges with $10 \mathrm{~mm}$ base attached in both longitudinal (direction of maximum normal stress) and transverse directions (indicated no strain). To increase accuracy the Young's modules $E$ were found in two ways:

$$
E^{1}=\frac{P l}{4 \varepsilon W} \text { and } E^{2}=\frac{P l^{3}}{48 \Delta J} .
$$

Additionally, acoustic emission was monitored by one sensor attached to CC surface. Absence of acoustic emission verified that tests were conducted by only elastic deformation and approved the Hook's law application.

Descent rate $n$ was measured by free oscillation graph recorded from vibration sensor attached to the center of $\mathrm{CC}$ and calculated by formula:

$$
n=\frac{1}{T} \cdot \ln \frac{A(t)}{A(t+T)},
$$

where: $A(t)$ and $A(t+T)$ - oscillation amplitudes measured in time $t$ and $t+T$ correspondently, $T-$ oscillation period.

The experimental research was conducted for the circuit card with DFD structure of type 1 (fig. 5, a). The tested assembly was fixed on the shaker by the rigid clamps.

Physical and mechanical parameters of both CC and DFD determined by method of testing parameters are represented in tables 1,2 .

Table 1 Parameters of circuit card.

\begin{tabular}{|l|l|l|l|l|l|l|}
\hline $\begin{array}{l}\text { Dimensions, } \\
\mathrm{mm}\end{array}$ & $\begin{array}{l}\text { Substrate } \\
\text { material }\end{array}$ & $\begin{array}{l}E_{1}, \\
\mathrm{GPa}\end{array}$ & $\begin{array}{l}\text { Substrate } \\
\text { ultimate } \\
\text { strength, MPa }\end{array}$ & $\begin{array}{l}\text { Solder joint } \\
\text { ultimate } \\
\text { strength, MPa }\end{array}$ & $\begin{array}{l}n_{1}, \\
1 / \mathrm{s}\end{array}$ & $\begin{array}{l}\mathrm{m}, \\
\mathrm{kg}\end{array}$ \\
\hline $180 \times 50 \times 1.5$ & $\begin{array}{l}\text { Fiberglass } \\
\text { CAST-V }\end{array}$ & 14 & $160-300$ & 40 & 5.48 & 0.05 \\
\hline
\end{tabular}


Table 2 Parameters of dry friction damper.

\begin{tabular}{|l|l|l|l|}
\hline Material & $E_{2}, \mathrm{GPa}$ & $n_{2}, 1 / \mathrm{c}$ & $\mu$ \\
\hline Carbon steel & 200 & 0.1 & 0.2 \\
\hline
\end{tabular}

Minimal threshold value for the stiffness of DFD $k_{\min }=718.75 \mathrm{~N} / \mathrm{m}$ calculated by $(21)$ over actual parameters given in (tables 1,2 ) was used to specify geometric parameters for DFD design introduced by the curved shape with rectangular cross-section: $50 \times 10 \times 1 \mathrm{~mm}$.

Vibration tests were conducted by constant dynamic force with amplitude $P_{0}$ provided by constant vibration acceleration $a_{0}=10 \mathrm{~m} / \mathrm{s}^{2}$. Maximal normal stress was determined by reading strain gauge attached to the middle point of CC surface and calculation by the Hook's law.

Maximal deflection was measured by vibration sensor set in the center of CC. the second vibration sensor attached to the fixtures on shaker was reading oscillations generated by the shaker. The resonance frequencies were detected by method of floating frequency $[11,12]$. The first critical frequency was detected by the sensor in the middle of CC. Stress measured on this frequency is considered as maximal dynamic stress.

Averaged results of 5 vibration tests and mathematical modeling of maximal dynamic stress and deflection in circuit card with DFD and without are given in table 3.

Table 3 - Maximal dynamic stress and deflection in circuit cards with DFD and without in the first critical frequency.

\begin{tabular}{|c|c|c|c|}
\hline \multirow{2}{*}{ Objects } & $\omega_{0}, \mathrm{rad} / \mathrm{s}$ & $\sigma_{d y n}^{\max }, \mathrm{MPa}$ & \multirow{2}{*}{$\Delta_{d y n}^{\max }, \mathrm{mm}$} \\
\hline \multirow{3}{*}{$\begin{array}{c}\text { Circuit card } \\
\text { with } \\
\text { DFD }\end{array}$} & 198.5 & \multicolumn{3}{|c|}{ Experimental data } \\
\cline { 2 - 4 } & \multicolumn{3}{|c|}{ Mathematical modeling data } \\
\cline { 2 - 4 } & 216.29 & 16.00 & 3.52 \\
\hline \multirow{3}{*}{ Circuit card } & 163.1 & \multicolumn{3}{|c|}{ Mathematical modeling data } \\
\cline { 2 - 4 } & 180 & \multicolumn{3}{|c|}{19.71} & 4.75 \\
\cline { 2 - 3 } & \multicolumn{3}{|c|}{5.07} \\
\cline { 2 - 4 }
\end{tabular}

Comparing maximal magnitudes of dynamic stress and deflection obtained by experimental tests with results of mathematical modeling indicates their similarity and insignificant relative difference from $-6.1 \%$ to $-16.8 \%$, what verifies sufficient accuracy of mathematical modeling $(19,20)$ where actual difference in calculation can be used as safety factor in engineering calculations.

Comparing maximal dynamic stress and deflection obtained by experimental tests of CCs with DFD and without indicated essential reduction of mentioned parameters by $20.5 \%$ and $25.9 \%$ correspondently and verified effectiveness of DFD to reduce maximal dynamic stress and deflection in critical section of circuit cards in conditions of inertial resonance excitation.

\section{Conclusions}

Method for reducing dynamic stress and deflection in critical section of circuit card in resonance condition has been developed by inserting additional elastic and dissipation joint introduced by dry friction damper, which effectiveness to reduce maximal dynamic stress and deflection is provided by its elastic and dissipation properties, in particular by its stiffness. The stiffness of dry friction damper is directly proportional to generalized elastic 
resistance force, it produces, which consists of elastic resistance force producing pressure of dry friction damper onto the clutch and friction force that resists damper sliding in the clutch. Increasing mentioned forces with increasing amplitude of vibration provides reducing dynamic stress and deflection transmitted to circuit card.

The mathematical model developed for maximal dynamic stress and deflection calculation in critical section of circuit card, which is represented by two-supported weightless prismatic beam with single concentrated mass joined to dry friction damper, in resonance condition and functional dependencies obtained on its stiffness demonstrate reduction of dynamic stress and deflection due to increasing stiffness of the dry friction damper. Mathematical model provides calculation of minimal threshold value for stiffness of dry friction damper, which ensures compliance with acceptable strength standard, and on this basis design specification of its geometric parameters.

Verification of sufficient accuracy of stiffness minimal threshold value estimation for the dry friction damper and its effectiveness to reduce maximal dynamic stress and deflection in critical section of circuit cards in conditions of inertial resonance excitation has been obtained by comparing results of mathematical modeling and experimental research of circuit cards with dry friction damper.

\section{References}

1. H.Xie, Q.Tan, Y.C.Lee, Y. Goh, Electronic Packaging: Thermal, Mechanical, and Environmental Durability. Reference Module in Materials Science and Materials Engineering, (Elsevier BV, 2018), https://doi.org/10.1016/B978-0-12-803581-8.020269

2. Y.J. Wan, G. Li, Y.M. Yao, X.L. Zeng, P. L. Zhu, R. Sun, Recent advances in polymerbased electronic packaging materials. Composites Communications, 19, 154-167 (2020), https://doi.org/10.1016/j.coco.2020.03.011

3. N. Muhammad, Z. Fang, M. Shoaib, Remaining useful life (RUL) estimation of electronic solder joints in rugged environment under random vibration. Microelectronics Reliability, 107, 113614 (2020), https://doi.org/10.1016/j.microrel.2020.113614

4. J. Franke, L. Wang, K. Bock, J. Wilde, Electronic module assembly. CIRP Annals, 70(2), 471-493 (2021), https://doi.org/10.1016/j.cirp.2021.05.005

5. E. Suhir, Analytical ("mathematical") modeling of the dynamic response of a printed circuit board (PCB) to an impact load, in Proceedings of the 2010 IEEE 11th International Conference on Electronic Packaging Technology \& High Density Packaging, 16-19 Aug. 2010, Xi'an, China, 480-488 (2010) https://doi.org/10.1109/ICEPT.2010.5583788

6. T.Y. Park, H.U. Oh, New PCB strain-based structural design methodology for reliable and rapid evaluation of spaceborne electronics under random vibration. International Journal of Fatigue, 146, 106147 (2021), https://doi.org/10.1016/j.ijfatigue.2021.106147

7. V.N. Somashekar, S.Harikrishnan, P.S.M. Aejaz Ahmed, D.Kamesha, Vibration Response Prediction of the Printed Circuit Boards Using Experimentally Validated Finite Element Model. Procedia Engineering, 144, 576-583 (2016), https://doi.org/10.1016/j.proeng.2016.05.044

8. P. Rajaguru, H. Lu, C. Bailey, M. Bella, Modelling and analysis of vibration on power electronic module structure and application of model order reduction. Microelectronics Reliability, 110, 113697 (2020), https://doi.org/10.1016/j.microrel.2020.113697 
9. P. Veeramuthuvel, K.K.Sairajan, K.Shankar, Vibration suppression of printed circuit boards using an external particle damper. Journal of Sound and Vibration, 366, 98-116 (2016), https://doi.org/10.1016/j.jsv.2015.12.034

10. H.A. El-Gohary, W.A.A.El-Ganaini, Vibration suppression of a dynamical system to multi-parametric excitations via time-delay absorber. Applied Mathematical Modelling, 36(1), 35-45 (2012), https://doi.org/10.1016/j.apm.2011.05.034

11. I. I. Kovtun, J. M. Boiko, S. A. Petrashchuk, Mathematical model for dynamic force analysis of printed circuit boards. Journal of Physics: Conference Series, 1921(1), 012120 (2021), https://doi.org/10.1088/1742-6596/1921/1/012120

12. I. Kovtun, J. Boiko, S. Petrashchuk, T. Kałaczyński, Theory and practice of vibration analysis in electronic packages. MATEC Web of Conferences, 182, 02015 (2018), https://doi.org/10.1051/matecconf/201818202015

13. I. Kovtun, J. Boiko, S. Petrashchuk, T. Kałaczyński, Methods for Vibration Reduction in Enclosed Electronic Packages. MATEC Web of Conferences, 302, 01008 (2019), https://doi.org/10.1051/matecconf/201930201008.

14. G.S. Pisarenko, V.A. Agarev. Strength of materials. (Kiev: Technika, 1967)

15. I. Kovtun, J. Boiko, S. Petrashchuk, G. Bauriene, K. Pilkauskas, Effects of the strain transmission from the main board to the installed electronic components. Mechanika, 22(6), 489-494 (2016), http://dx.doi.org/10.5755/j01.mech.22.6.16891

16. I. Kovtun, V. Royzman, A. Voznyak, Acoustic emission diagnostics of solder joints on printed circuit boards. Quality and Reliability of Technical Systems: Theory and Practice (JVE Book Series on Vibroengineering. Vol. 2. JVE International Ltd., Vilnius, Lithuania. 2018) 214-229. ISSN 2351-5260

17. I. Kovtun, V. Royzman, Stress computation and reduction by cyber-physical systems controlling printed circuit board manufacturing technology. Cyber-Physical Systems: Design and Application for Industry 4.0, Studies in Systems, Decision and Control, 342, 49-60, 2021, ISSN: 2198-4182I, https://doi.org/10.1007/978-3-030-66081-9_4

18. I. Kovtun, J. Boiko, S. Petrashchuk, Assessing Enclosure Case Design on Excitation and Transmission of Vibration in Electronic Packages, in Proceedings of the 2019 IEEE 2nd Ukraine Conference on Electrical and Computer Engineering, UKRCON, Lviv, Ukraine, 265-270, https://doi.org/10.1109/UKRCON.2019.8879782

19. P. Veeramuthuvel, K.K.Sairajan, K.Shankar, Application of particle damper on electronic packages for spacecraft. Acta Astronautica, 127, 260-270 (2016), https://doi.org/10.1016/j.actaastro.2016.06.003

20. V. Nekhaev, V. Nikolaev, A. Rauba, On the issue of effective protection of objects from vibration and shock. MATEC Web of Conferences, 239, 01053 (2018), https://doi.org/10.1051/matecconf/201823901053

21. M. Barys, R. Zalewski, Analysis of inertial amplification mechanism with smart springdamper for attenuation of beam vibrations, MATEC Web of Conferences, 157, 03002 (2018), https://doi.org/10.1051/matecconf/201815703002

22. T.Y. Park, S.J. Shin, S.W. Park, S.J. Kang, H.U. Oh, High-damping PCB implemented by multi-layered viscoelastic acrylic tapes for use of wedge lock applications Engineering Fracture Mechanics, 241, (2021) https://doi.org/10.1016/j.engfracmech.2020.107370

23. J. Boiko, I. Kovtun, S. Petrashchuk, Vibration transmission in electronic packages having structurally complex design, in Proceedings of the 2017 IEEE First Ukraine Conference on Electrical and Computer Engineering, UKRCON, 29 May-2 June 2017, Kyiv, Ukraine, 514-517, https://doi.org/10.1109/UKRCON.2017.8100294 